\title{
Garment embedded sweat-activated batteries in wearable electronics for continuous sweat monitoring
}

Xingcan Huang $\mathbb{D}^{1,3}$, Yiming Liu ${ }^{1,3}$, Jingkun Zhou $\mathbb{D}^{1,2,3}$, Sina Khazaee Nejad ${ }^{1,2}$, Tsz Hung Wong ${ }^{1}$, Ya Huang ${ }^{1,2}$, Hu Li ${ }^{1}$, Chun Ki Yiu ${ }^{1,2}$,

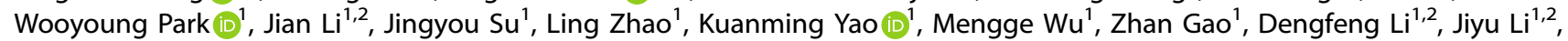
Rui Shi ${ }^{1}$ and Xinge Yu (iD ${ }^{1,2 凶}$

Thin, soft, and skin-integrated electronic system has great advantages for realizing continuous human healthcare monitoring. Here, we report an ultra-thin, flexible, and garment-based microelectronics powered by sweat-activated batteries (SABs) and applications of powering biosensors and microelectronic systems for real time sweat monitoring. The $S A B$ cell is ultra-thin $(1.25 \mathrm{~mm})$ with excellent biocompatibility. The $\mathrm{SAB}$ has good electricity output with high capacity (14.33 mAh) and maximum power density $\left(3.17 \mathrm{~mW} \mathrm{~cm}^{-2}\right)$ after being activated by the sweat volume of $0.045 \mathrm{~mL} \mathrm{~cm}^{-2}$, which could continuously power 120 light emitting diodes over $3 \mathrm{~h}$. The outputs could maintain stable after repeatable bending. Wireless microelectronics system could be continuously powered by the SABs for $3 \mathrm{~h}$ to monitor sweat and physiological information, including sweat $\mathrm{Na}^{+}$concentration, $\mathrm{pH}$, and skin impedance. The reported integrated system provides a potential for solving the power issues of flexible wearable electronics and realizing personalized medicine.

npj Flexible Electronics (2022)6:10; https://doi.org/10.1038/s41528-022-00144-0

\section{INTRODUCTION}

Wearable devices with the advances in small size, light weight, and on body features, associating with flexible electronic technologies have attracted great attention in the fields of healthcare monitoring, entertainment, and human-machine interfaces $^{1-10}$. Human body, as a remarkable biological system with complex organized physiological processes, associates with lots of biochemical and biophysical signals that could reflect the health status $^{6,11,12}$. Developing wearable sensors is extremely essential to realize the personalized on-patient healthcare because they can continuously monitor individuals' physiological biomarkers. Sweat is a typical body fluid containing information of multiple biomarkers such as $\mathrm{pH}$, inorganic ion concentration, and moisture, that can be used as natural testing samples in daily life, since changes in the concentration of these biomarkers are closely related with human physical condition ${ }^{13-15}$. The traditional sweat testing methods associate with collecting sweat samples from skin by gauze pads and then analyzed by benchtop instrument, which is time consuming and may cause the sample pollution ${ }^{16,17}$. Recent advances in wearable sweat sensors highlights the importance of this field ${ }^{18-22}$. While many efforts have been focused on developing sweat sensors with high sensitivity and wearing comfortability. There are still some critical issues needed to be addressed for wearable sweat monitoring systems, such as portable power sources and data transmission modules.

Power source as the most important component in wearable electronics; however, is still an obstacle restricting the miniaturization, flexibility, and integration of wearable electronics. Up to now, the most common used powering strategies for wearable devices mainly rely on (a) energy storage components, i.e., lithium-ion battery and super-capacitor ${ }^{23-29}$, and (b) energy harvesting and self-powered devices, such as triboelectric nanogenerators, solar cells, and biofuel cells ${ }^{30-38}$. For the energy storage components, batteries and super-capacitors are typically based on rigid and bulky devices, which is not appropriate for wearable electronics. In addition, the electrolytes of commercial rechargeable batteries are strong acid or base, which raises safety concerns during usage. For self-powered devices, the electrical outputs are too low to power and operate microcontrollers for achieving the continuous data collection, processing, and transmission. These devices typically require supercapacitors as energy storage for intermittent operation. Very recently, the concept of sweat-activated battery $(\mathrm{SAB})$ and demonstrations in soft electronics offer a new strategy for power management in wearable electronics for continuous sweat monitoring ${ }^{17,39}$. Nevertheless, there are still some problems need to be solved for SABs, especially the dimension in thickness, flexibility, and power outputs.

Here, we report a class of materials and devices of wearable sweating sensing electronic system based on ultra-thin, soft, skinintegrated $S A B$ and state of art flexible sweat sensors. The reported $S A B$ could produce sufficient electricity to power the microcontroller (MCU) for continuously data acquisition, processing, and storage of multiplexed monitoring of key metabolic biomarkers in sweat (e.g., sodium ion $\left(\mathrm{Na}^{+}\right.$) concentration, $\mathrm{pH}$ values, and skin impedance) in a wireless data transmission way, and thus allows intelligent dehydration prevention and health monitoring during exercise. The $S A B$ unit cell $(3.5 \mathrm{~cm} \times 1 \mathrm{~cm})$ with optimized architecture, materials, and mechanics designs exhibits ultra-thin in thickness of $1.25 \mathrm{~mm}$, great outputs with an open circuit voltage of $1.54 \mathrm{~V}$ and a short circuit current of $16.91 \mathrm{~mA}$, and a capacity of $14.33 \mathrm{mAh}$ and a maximum power density (MPD) of $3.17 \mathrm{~mW} \mathrm{~cm}^{-2}$, that could continuously light 120 light-emitting diodes (LEDs) over $3 \mathrm{~h}$. Excellent flexibility of the SAB allows stable and robust performance under mechanical deformations, with no performance degradation over hundreds of cycles bending at $180^{\circ}$. The demonstration of the SAB associates with powering a multiplexed sweat sensors patch and a near field communication

${ }^{1}$ Department of Biomedical Engineering, City University of Hong Kong, Hong Kong 999077, China. ${ }^{2}$ Hong Kong Center for Cerebra-Cardiovascular Health Engineering, Hong Kong Science Park, New Territories, Hong Kong, China. ${ }^{3}$ These authors contributed equally: Xingcan Huang, Yiming Liu, Jingkun Zhou. ${ }^{凶}$ email: xingeyu@cityu.edu.hk 

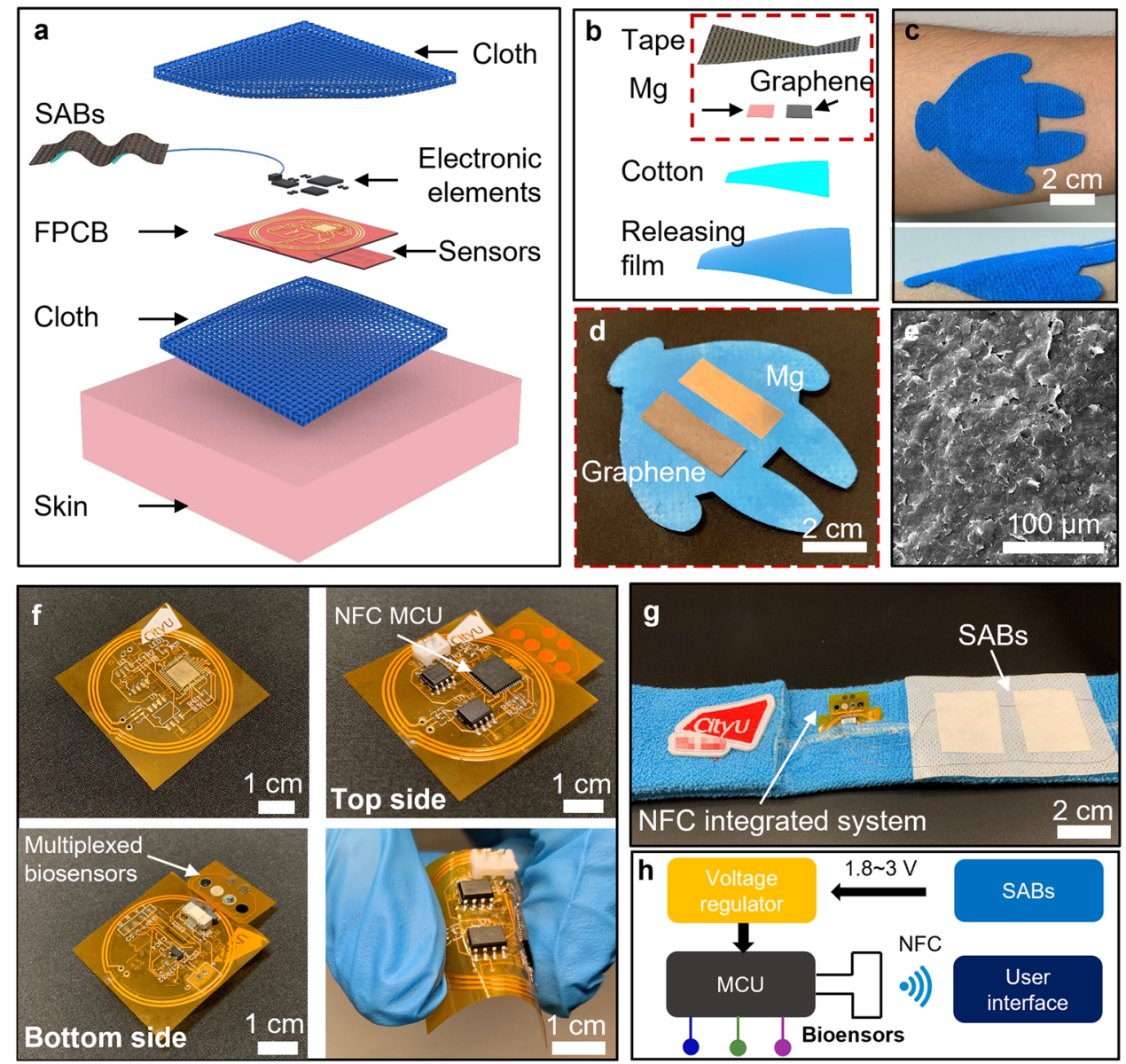

Fig. 1 SABs powered microelectronics system for multiplexed wireless sensing. a Schematic illustrations of the skin integrated electronic system. $\mathbf{b}$ Schematic illustrations of the ultra-thin SAB. c Optical images of the SAB mounted on human forearm and the enlarged details of side views with the Cartoon shape of a Baymax. d Optical images of the electrodes for the SAB. e SEM image of graphene-coated absorbent paper. $\mathbf{f}$ Optical images of the microelectronics system integrated with multiplexed biosensor patch and flexible circuit. $\mathbf{g}$ Optical images of the microelectronics system embedded into a head band. $\mathbf{h}$ System-level block diagram of the electronic module for the whole system.

(NFC) based microelectronics for continuous monitoring sweat and skin impedance for $3 \mathrm{~h}$, indicating the possibility of next generation self-powered wearable sweat sensors.

\section{RESULTS}

\section{SABs powered microelectronics system for wireless sensing}

Figure 1a shows the schematic illustrations of the SAB-powered flexible microelectronics system (SMS), which consists of an ultrathin powering components based on SABs, and a flexible sweating sensing devices with the integration of biosensors for physiological related information detection in sweat and a NFC-based wireless microelectronic device for data collection, processing, and wireless communications. The thin, soft features of the $S A B$ and NFC electronic sensing device allow the SMS to be easily integrated with cloth and thus to be attached onto various parts of body in wearable formats, such as headbands sport braces to wear on heads, elbows, and knees for sweat monitoring. Considering the biocompatibility and performance issues, magnesium (Mg)/oxygen $\left(\mathrm{O}_{2}\right)$ based reaction principle was selected as the powering option for the SABs, as which is a kind of emerging green power sources with considerable capacity, low cost, and good biocompatibility ${ }^{40-42}$. Figure $1 \mathrm{~b}$ shows the schematic diagram of the ultra-thin, flexible $S A B$, which is comprised by a porous tape with releasing layer (thickness, $0.3 \mathrm{~mm}$ ) for good air breathing and immobilizing inner components, a Graphene coated paper (size, $1 \mathrm{~cm} \times 3 \mathrm{~cm}$; thickness, $0.15 \mathrm{~mm}$ ) as the cathode, a thin $\mathrm{Mg}$ foil (size, $1 \mathrm{~cm} \times 3 \mathrm{~cm}$; thickness, $0.09 \mathrm{~mm}$ ) as the anode, a thin cotton layer (thickness, $0.8 \mathrm{~mm}$ ) with potassium chloride $(\mathrm{KCl})$ powders insides serving as rapid sweat absorption layer and thus serves as electrolyte. Noted, the materials selected in the SABs are all low-cost and safe to skin, and environmental friendly. After collecting sweat in the cotton layer and forming liquid environment, $\mathrm{Mg}$ foil could react with $\mathrm{O}_{2}$ and water $\left(\mathrm{H}_{2} \mathrm{O}\right)$ to produce magnesium hydrate $\left(\mathrm{Mg}(\mathrm{OH})_{2}\right)$ (Eq. 1). Therein, the $\mathrm{Mg}$ anode could be oxidized and release electrons (Eq. 2). At the same time, $\mathrm{O}_{2}$ could be reduced in the Graphene cathode and obtain electrons (Eq. 3). As a result, an electron transport circuit could be formed between the anode and cathode. Here, for improving the output performance of the $S A B$, the graphenecoated absorbent paper is utilized for catalyzing oxygen reduction and adsorbing electrolyte ${ }^{43-45}$.

Figure 1e shows the scanning electron microscopy (SEM) images of the Graphene coated absorbent paper, where adequate Graphene flakes can be found on the paper. Compared with the neat absorbent paper without any materials coating (Supplementary Fig. 1), it is obvious that graphene has been evenly covered on the surface of the paper, which could significantly enhance the catalytic efficiency. The target of the system is to integrate with cloth, and the format of the SAB should be in an ultrathin architecture, therefore we adopted an in-plane structure for the $S A B$ where the anode and cathode are designed as the side by side structure (Fig. 1d and Supplementary Fig. 2). As demonstrated in Fig. 1c, the ultra-thin format (overall thickness, $1.25 \mathrm{~mm}$ ), 
lightweight $(0.65 \mathrm{~g})$, and great flexibility allows the SAB convenient to interface with human skin for long-term use and also could be designed as various expected shapes for aesthetics. Supplementary Fig. 3 shows the good biocompatibility of the SAB as there is not any skin irritation reaction after wearing $3 \mathrm{~h}$. The $S A B$ could be stored in vacuum seal bags before using, that effectively prevent the performance degradation caused by the active chemical property of Mg (Supplementary Fig. 4).

Overall: $\mathrm{Mg}+\mathrm{O}_{2}+2 \mathrm{H}_{2} \mathrm{O} \rightarrow 2 \mathrm{Mg}(\mathrm{OH})_{2}$

Anode $: \mathrm{Mg} \rightarrow \mathrm{Mg}^{2+}+2 e^{-}$

Cathode : $\mathrm{O}_{2}+2 \mathrm{H}_{2} \mathrm{O}+4 e^{-} \rightarrow 4 \mathrm{OH}^{-}$

The SMS was designed by well-established flexible electronics technologies ${ }^{46-48}$, which is compatible with flexible print circuit board (FPCB) manufacturing processes, and thus capable of achieving high reliability and low cost manufacture. As shown in Fig. 1f, the integrated SMS including the detachable multiplexed biosensor patch, an NFC chip, and electronic components is built on an ultra-soft and thin polyimide ( $\mathrm{PI}$, $130 \mu \mathrm{m})$ substrate, that offers excellent flexibility with stable performance under bending at a board range of angles, and thus could be entirely embedded into cloth and affixed to the skin for sweat monitoring during exercise (Fig. 1g). A small amount of sweat generated by a short time of activities would activate the SAB for powering the wearable SMS to continuously monitor biomarkers of $\mathrm{Na}^{+}$concentration, $\mathrm{pH}$ values of sweat as well as the skin impedance. The NFC-based MCU is used for data acquisition, processing, and storage. The wireless data transmission happened when a smartphone approaches the SMS, where the stored data of the continuous monitored period of time could be displayed on the graphic user interface (GUI) of the smartphone (Fig. 1h).

\section{Performance characterization of the SABs}

Figure 2 shows the electrical characteristics of the $S A B$ as functions of various several parameters, including distance between cathode and anode (Fig. 2a), thickness of the Mg foil (Fig. 2b), and ambient temperature (Fig. 2c), which are directly associating with the device size, operation time and output performance. It is found that longer distance between the electrodes (Fig. 2a) and thinner Mg foil (Fig. 2b) tend to decrease the operation duration of the SAB with smaller average short-circuit current. At a constant $\mathrm{Mg}$ foil thickness of $90 \mu \mathrm{m}$ and ambient temperature of $25^{\circ} \mathrm{C}$, the working duration of the $S A B$ and the corresponding average short-circuit current drops from $3.52 \mathrm{~h}$ and $14.98 \mathrm{~mA}$ as the distance between electrodes increases from 0.5 to $2.5 \mathrm{~cm}$, where the result is owing to the increase of the inner impedance of the battery (Fig. 2a). From the short-circuit current versus $\mathrm{Mg}$ foil thickness shown in Fig. $2 \mathrm{~b}$, it can be found that thicker $\mathrm{Mg}$ electrode contributes positive effect on the duration and shortcircuit current. Specifically, SABs with $30 \mu \mathrm{m}$ thick Mg electrode yield operating duration of $0.73 \mathrm{~h}$ with average short-circuit current of $16.24 \mathrm{~mA}$, while SABs with $90 \mu \mathrm{m}$ thick Mg electrode exhibits the performance of $3.52 \mathrm{~h}$ and $16.91 \mathrm{~mA}$. As a result, the performance of the SABs can be simply optimized by adjusting the distance between electrodes and $\mathrm{Mg}$ electrode thickness.

Next, we measured the discharging capacity of the SABs and the influences of the electrolyte and ambient temperatures on battery capacity. Supplementary Fig. 5 shows the capacity of the SABs can reach to $14.33 \mathrm{mAh}$ when $\mathrm{KCl}$ is used as the electrolyte and salt bridge. Other electrolyte materials including magnesium chloride $\left(\mathrm{MgCl}_{2}\right)$ and calcium chloride $\left(\mathrm{CaCl}_{2}\right)$ were also adopted to compare the $\mathrm{SAB}$ performance, where the capacity of the SABs using $\mathrm{MgCl}_{2}$ and $\mathrm{CaCl}_{2}$ as electrolyte was only $2.99 \mathrm{mAh}$ and 1.47 $\mathrm{mAh}$, demonstrating $\mathrm{KCl}$ electrolyte is the best candidate in this type of SABs. Figure $2 c$ shows the capacity differences of the SABs under different ambient temperatures, where it can be found the energy capacity decreases with the increase of ambient temperature from $14.33 \mathrm{mAh}$ at $25^{\circ} \mathrm{C}$ to $6.82 \mathrm{mAh}$ at $45^{\circ} \mathrm{C}$. The results can be explained as the increases of evaporation rates under lowflow-rate conditions, resulting in increased internal resistance (Fig. $2 \mathrm{c})^{17,49}$. Figure $2 \mathrm{~d}$ shows the open-circuit voltage as a function of time of a representative SAB cell after absorbing sweat, where the voltage can stabilize at $1.54 \mathrm{~V}$ for over $5 \mathrm{~h}$. The polarization data shown in Fig. 2e shows that the maximum current density (MCD) could reach $3.88 \mathrm{~mA} \mathrm{~cm}^{-2}$. Accordingly, the MPD is calculated as $3.17 \mathrm{~mW} \mathrm{~cm}^{-2}$ (Fig. 2f). To investigate the influence of the absorbed sweat volume on the electrical performance of the SABs, we measured the open-circuit voltage of the batteries by gradually increasing artificial sweat volume from 0 to $0.045 \mathrm{~mL} \mathrm{~cm}^{-2}$, as shown in Fig. $2 \mathrm{~g}$. It is obvious that the battery can be activated with a very small amount of sweat volume of $0.021 \mathrm{~mL} \mathrm{~cm}^{-2}$, with an open-circuit voltage of $0.59 \mathrm{~V}$. This small amount of sweat can be easily collected during daily activities, which allows our SABs to exhibit an extremely short activation time (30 s) during activities (Supplementary Table 1). Benefited from the advances in mechanics, the SABs are capable of maintaining stabilized opencircuit voltage around $1.52 \mathrm{~V}$ with negligible fluctuations under bending in a broad range from $90^{\circ}$ to $180^{\circ}$ (Fig. 2h). In addition, the voltage outputs of the SABs stabilize between 1.45 and $1.59 \mathrm{~V}$ during repeatable bending test, and exhibit negligible degradation after 600 bending cycles at a constant angle and frequency of $90^{\circ}$ and $2.8 \mathrm{~Hz}$ (Fig. 2i).

\section{Multiplexed biosensor patch characterization}

To verify the performance of the SABs, a load of a 120 green LEDs array was connected to a SAB cell activated by $0.135 \mathrm{~mL}$ to test the lighting duration, where it came out the LED array can be continuously lighted over $3 \mathrm{~h}$ with high brightness, demonstrating the great capacity and output energy of the SABs (Supplementary Fig. 6 and Supplementary Movie). Encouraged by the excellent performance of the SABs, we next integrated the SABs with the SMS to power multiplexed biosensors for garment-embedded electronics to monitor sweat during exercise (Fig. 3). Figure 3a shows the sensor array with $\mathrm{Na}^{+}, \mathrm{pH}$ and impedance sensors we developed on PI substrate, where we can see the size of the integrated sensor array patch is very small, similar to the size of our fingertips. The $\mathrm{pH}$ sensor associates with the polyaniline (PANI) electrodeposited on the gold $(\mathrm{Au})$ electrode as the sensing layer. The $\mathrm{Na}^{+}$sensor adopts the method of ion-selective electrode (ISE) based on $\mathrm{Au}$ substrate for $\mathrm{Na}^{+}$concentration detection. The $\mathrm{pH}$ sensor shares the same corresponding silver (Ag)/silver chloride $(\mathrm{AgCl})$ reference electrode with the $\mathrm{Na}^{+}$sensor, and couples with a thin polyvinyl butyral (PVB) coating layer to maintain a stable potential in solutions with different ionic strengths. On the top of the sensor array patch, a pair of Au electrodes serves as the impedance sensor to get the skin impedance in a constant distance. Combining with the detachable multiplexed sensor patch, the integrated SMS could be easily embedded with sport wears, i.e., the head bands, elbow pads, and knee pads for sweat monitoring during exercise (Fig. 3b-d).

The performance of each type of sensor was characterized and optimized as shown in Fig. 3e-j. Supplementary Figs. 7 and 8 show the representative open circuit voltage responses of the $\mathrm{Na}^{+}$and $\mathrm{pH}$ sensors for the physiologically relevant concentrations of $10-100 \mathrm{mM} \mathrm{Na}^{+}$concentration and $2-8 \mathrm{pH}$ value, respectively ${ }^{50,51}$. The plots in Fig. 3e, f show good linear relationships between the voltage outputs and the logarithm of ion concentration for both the $\mathrm{Na}^{+}$sensors and $\mathrm{pH}$ sensors. The determination coefficient $\left(R^{2}\right)$ of $\mathrm{Na}^{+}$sensors and $\mathrm{pH}$ sensors could reach to 0.993 and 0.998 , respectively, indicating great sensitivities of these two biomarkers. Figure $3 \mathrm{~h}, \mathrm{i}$ shows the selectivity study results, and the data 
a

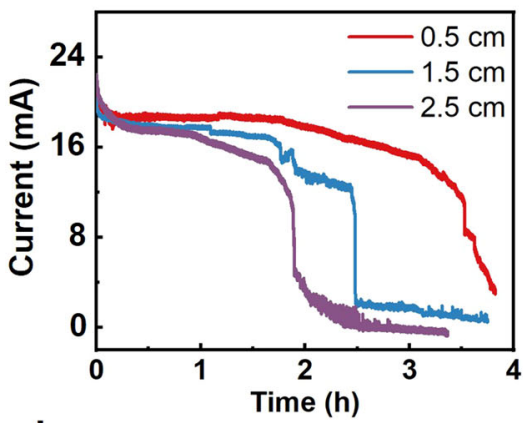

d

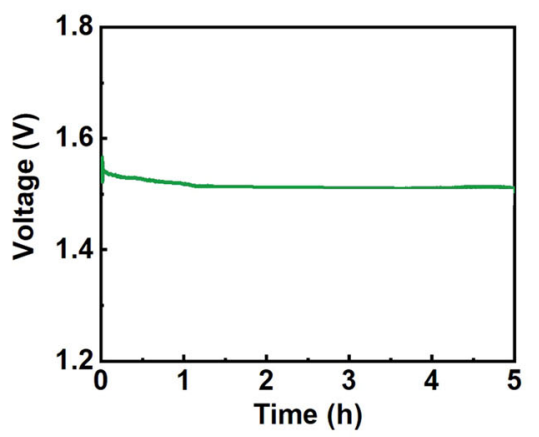

g

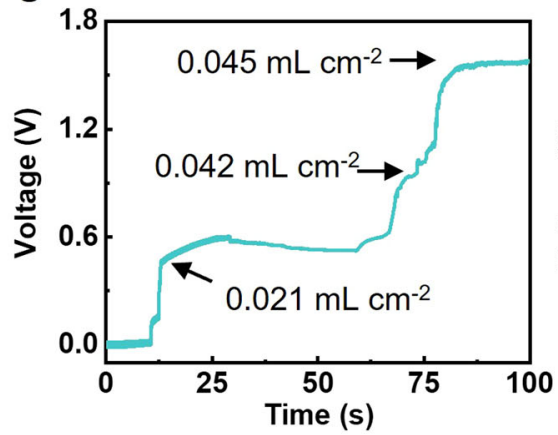

b

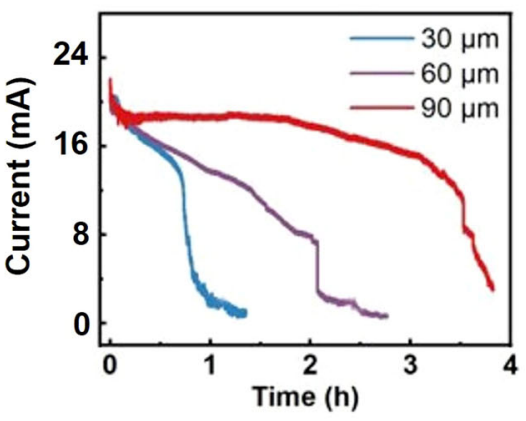

e

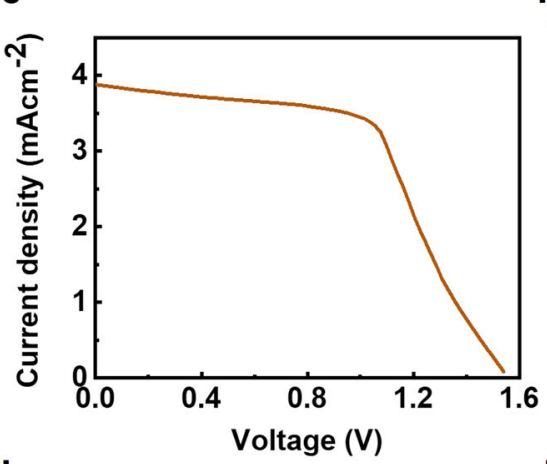

h

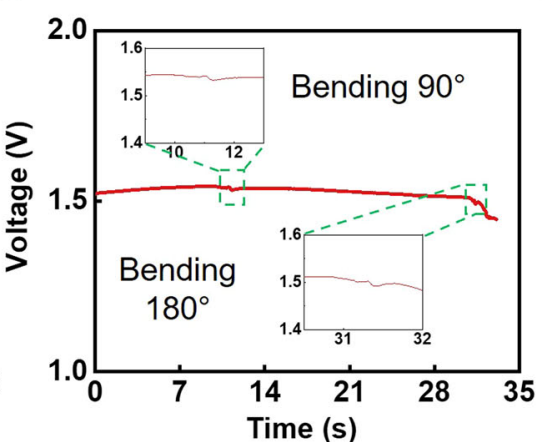

C

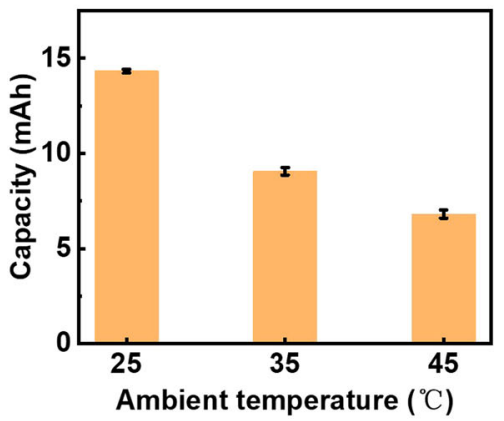

f

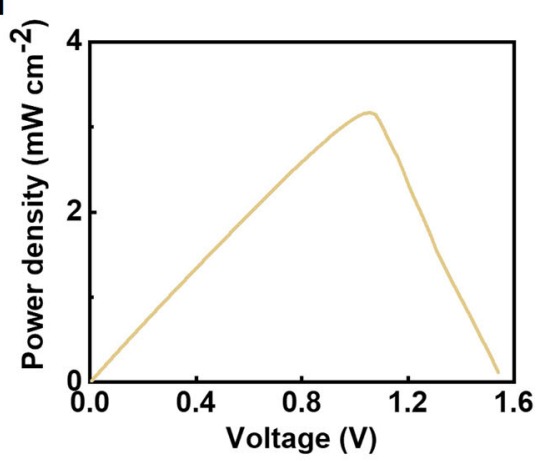

i

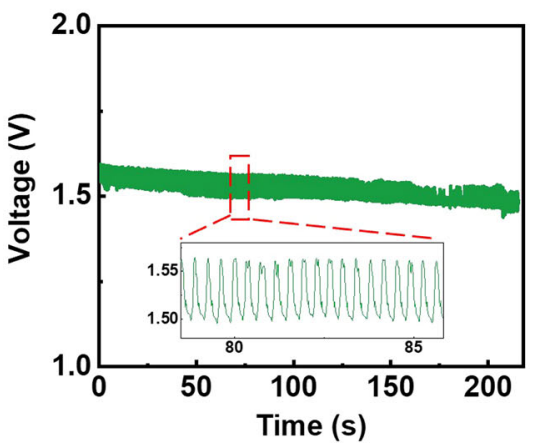

Fig. 2 Performance characterization of the SABs. a Short-circuit current of the SABs with three different electrode distances of 0.5, 1.5, and $2.5 \mathrm{~cm}$, as a function of time. b Short-circuit current of the SABs with three different $\mathrm{Mg}$ foil thickness, 30, 60, and $90 \mu \mathrm{m}$, as a function of time. c Influences of ambient temperature on the SABs' capacity. Discharging current, $1 \mathrm{~mA} \mathrm{~cm}{ }^{-2}$. d Open-circuit voltage of the SABs. e Polarization curve of the SABs. $\mathbf{f}$ Power density curve of the SABs. $\mathbf{g}$ Electrical response of the SABs as a function of time with increasing injected sweat volume. $\mathbf{h}$ Electrical output response of the SABs bended as $90^{\circ}$ and $180^{\circ}$ as a function of time. i Electrical output response of the SABs bendeing over 600 times as a function of time.

indicates excellent anti-interference ability of the $\mathrm{Na}^{+}$sensors and $\mathrm{pH}$ sensors. It is clear that the $\mathrm{Na}^{+}$sensor is not sensible for other cations besides $\mathrm{Na}^{+}$, as the introduction of additional potassium chloride $(\mathrm{KCl})$ and ammonium chloride $\left(\mathrm{NH}_{4} \mathrm{Cl}\right)$ doesn't cause any electrical responses, while obvious responses only happened as sodium chloride $(\mathrm{NaCl})$ added in, demonstrating the sensor exhibiting good specificity for $\mathrm{Na}^{+}$(Fig. 3h). Figure $3 i$ shows the anti-interference study of the $\mathrm{pH}$ sensors, where the study associates with adding neutral chemicals of $\mathrm{KCl}$ and $\mathrm{NaCl}$, as well acid step by step. We can find that adding $\mathrm{KCl}$ and $\mathrm{NaCl}$ wouldn't influence the voltage outputs of the $\mathrm{pH}$ sensor, while the sensor is only responsive to the change of $\mathrm{H}^{+}$concentration. The characterization of the impedance sensors associates with measuring the skin impedance changes with different hydration levels by simply smearing water. Figure $3 \mathrm{~g}$ shows the skin impedance measured by the impedance sensors could reasonably increase with the water evaporation, demonstrating the feasibility of this method to detect skin impedance. Moreover, a commercial skin hydration meter (Supplementary Fig. 9, Real Bubee Ltd. UK) is used to calibrate the skin impedance sensor, as the hydration value is inversely proportional to the impedance. Figure $3 \mathrm{j}$ exhibits the comparison results between commercial hydration meter and the impedance sensor, demonstrating that the skin impedance and hydration on different body locations are different, as well as the usability and stability of the impedance sensor.

\section{On-body real-time health monitoring and wireless data transmission}

With the sensitive, stable, and robust performance of the sensor array, as well as the great capacity of the $S A B$, we next conducted the system level test of the SMS to demonstrate the potential in practical applications. Figure 4a shows a testing subject wears a SMS embedded elbow pad and jogs. The enlarge image in Fig. $4 a$ clearly shows that the multiplexed sensor patch could perfectly 

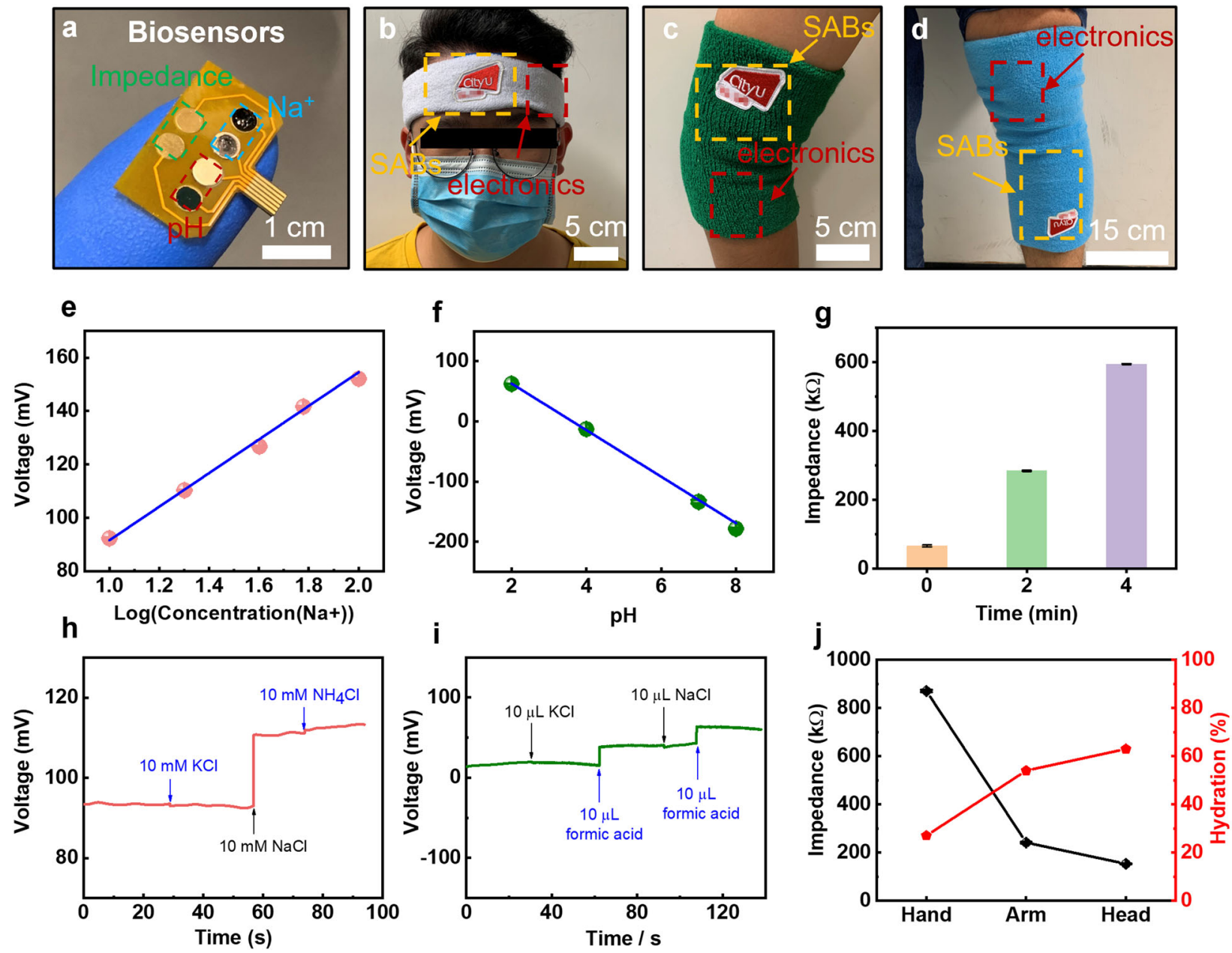

Fig. 3 Multiplexed biosensor patch characterization. a Optical images of the multiplexed biosensor patch. b-d Optical images of SMS embedded in the head band, elbow pad, and knee pad. e, $\mathbf{f}$ Calibration curve of the $\mathrm{Na}^{+}$sensor and pH sensor. $\mathbf{g}$ The skin impedance changes as a function of time after dropping $10 \mu \mathrm{L}$ water. $\mathbf{h}$, $\mathbf{i}$ Anti-interference characterization of the $\mathrm{Na}^{+}$sensor and pH sensor. $\mathbf{j}$ Results comparison of the skin impedance and commercial hydration instrument.

attach to the skin for sweat monitoring. When the subject begins to perspire, $2 S A B$ cells connected in series could be activated quickly and power the microelectronics system and sweat sensors. The signals obtained by the sensor patch would be converted to digital signals by 12-bit ADCs inside the MCU, and then these signals will be stored in a $2 \mathrm{M}$ electrically erasable programmable read-only memory (EEPROM) via I2C. When the phone is approaching, the NFC chip detects an electromagnetic field nearby, then it will send a signal to the MCU, so that the MCU will enter the interrupt, start reading the data stored in the EEPROM through the $12 \mathrm{C}$, and transfer these data to the phone through the NFC chip. The app in the smart phone will display and automatically store the received data for further analysis. Supplementary Fig. 10 demonstrates that the energy output by SABs could continuously support the normal work of the microelectronics system around $3 \mathrm{~h}$. Supplementary Fig. 11 shows the working stability of NFC-based microelectronics device. The normal peak frequency of the NFC system is $13.56 \mathrm{MHz}$, while that would shift to 13.53 and $13.495 \mathrm{MHz}$ when bended at $30^{\circ}$ and $60^{\circ}$. According to the NFC technical protocol of ISO 15693, the NFC communication system can work normally in the frequency range of $13.56 \pm 0.07 \mathrm{MHz}^{6,52,53}$. So, the NFC-based microelectronics device could transfer data normally during activities.

Continuous physiological monitoring is performed on a subject over $0.5 \mathrm{~h}$ jogging to demonstrate the real time monitoring concept. As shown in Fig. 4b, while the subject is jogging, the $\mathrm{Na}^{+}$ in sweat would increase at the beginning, and then stabilize over time because of the increase of sweat volume could dilute the concentration. There is minor fluctuation for the sweat $\mathrm{pH}$ ranging from 3.98 to 4.99 during exercising. As for the skin impedance, the impedance of the skin surface would keep decreasing because of the increase of skin moisture caused by perspiration, while the ion concentration in sweat has little effect on the change tendency of skin impedance. All the results demonstrate that the subject's physical condition stays stable at the early exercise stage, which is consistent with previous study results ${ }^{12,35,47,50}$. In Fig. $4 \mathrm{c}-\mathrm{e}$, the sweat $\mathrm{Na}^{+}$concentration, $\mathrm{pH}$, and skin impedance of three different subjects after continuous exercise are compared with the initial levels. It is clear that the sweat $\mathrm{Na}^{+}$concentration would increase a lot; the $\mathrm{pH}$ value slightly increase and impedance would decrease for every subject. Figure $4 \mathrm{f}-\mathrm{h}$ demonstrates changes of sweat composition and skin impedance in different body locations before and after exercise, which is also consistent with the change trends mentioned previous. These results all prove that general change trends of sweat and skin impedance caused by exercise as well as the feasibility and the stability of the SMS.

\section{DISCUSSION}

In summary, we developed an ultra-thin, flexible, and fully integrated flexible electronic system powered by SABs, that can be used for sweat monitoring in real time with wireless 
a

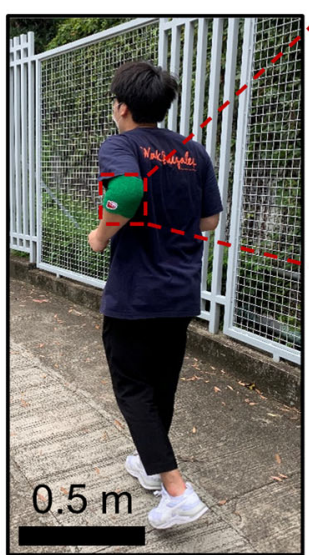

C

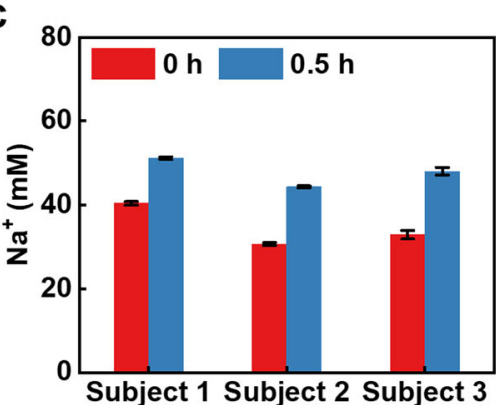

f

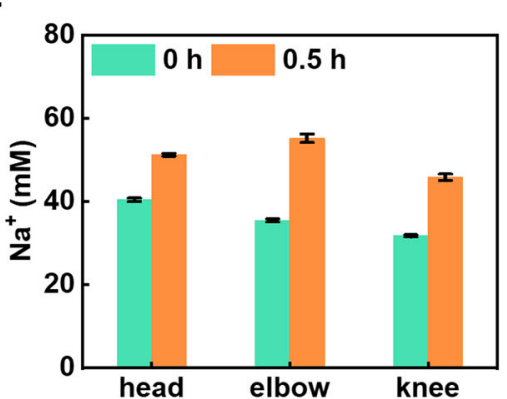

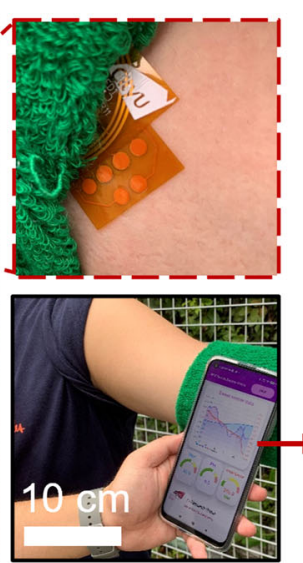

d

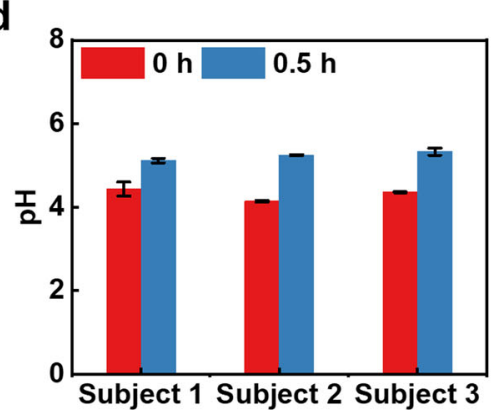

$g$

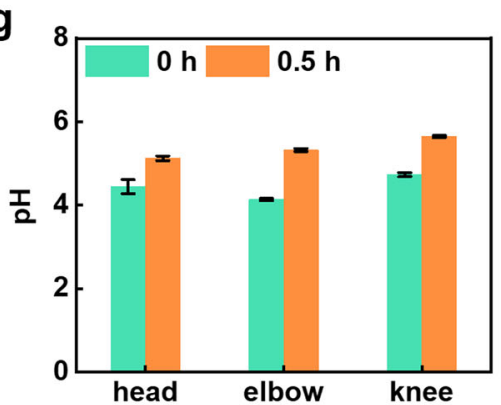

b

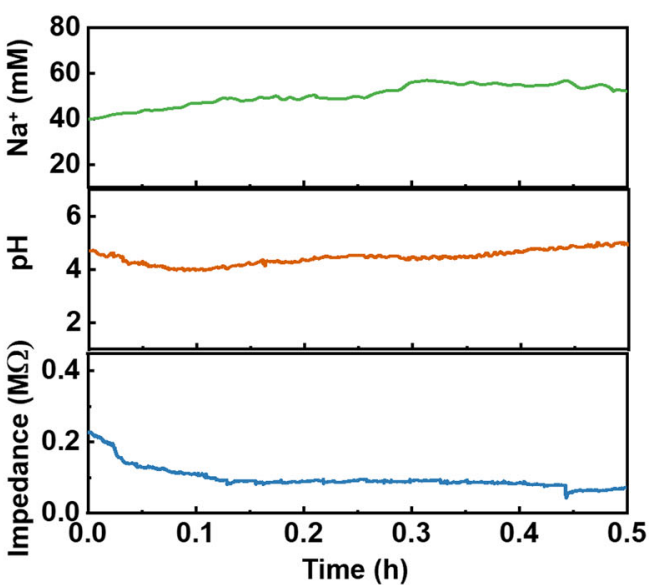

e

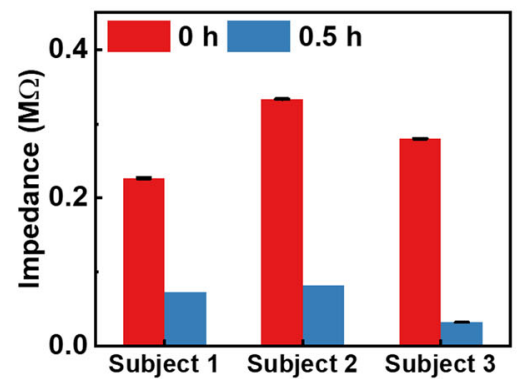

h

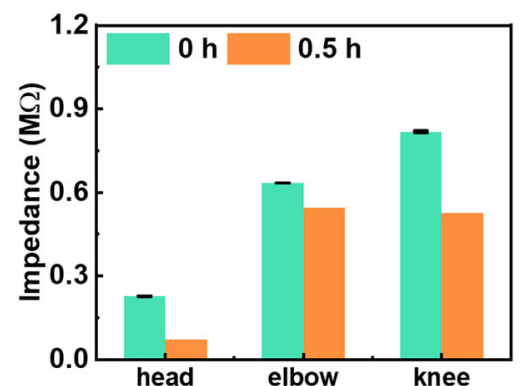

Fig. 4 On-body real-time health monitoring and wireless data transmission. a Optical images of the jogging subject wearing SMS embedded in the elbow pad and the phone interface that illustrates wireless communication and data acquisition. $\mathbf{b}$ The real-time monitoring results of the sweat $\mathrm{Na}^{+}$concentration, $\mathrm{pH}$ value and skin impedance during jogging. c-e The comparison of data changes of $\mathrm{Na}^{+}$ concentration, $\mathrm{pH}$ value, and skin impedance during exercise on heads of three different subjects. $\mathbf{f}-\mathbf{h}$ The comparison of data changes of $\mathrm{Na}^{+}$ concentration, $\mathrm{pH}$ value, and skin impedance during exercise for three different body locations, including head, elbow, and knee.

transmission capability. The ultra-thin SABs with high output power density and long duration could cling to the skin, be activated by a slight sweat volume, and light 120 LEDs over $3 \mathrm{~h}$. Flexible and sensitive biosensors are able to detect the sweat $\mathrm{Na}^{+}$ concentration, $\mathrm{pH}$ value, and skin impedance for dehydration prevention and healthcare monitoring during exercise. Powered by the SABs, signals of sensors could be continuously stored and processed by the microelectronics system, and wirelessly transmitted to the user interface by NFC. As wearable electronics, the whole SMS exhibits good conformal properties on skin surface. It can be assumed that the proposed integrated system would have wide application prospects in fields of wearable electronics and health monitoring.

\section{METHODS}

\section{Fabrication of SAB}

As exhibited in the Supplementary Fig. 12, the first step for fabricating SAB was to cut absorbent paper and $\mathrm{Mg}$ sheet into the fixed size $(3 \mathrm{~cm} \times 1 \mathrm{~cm})$.
And then immersing the obtained absorbent paper into graphene welldispersed aqueous solution and drying at $100^{\circ} \mathrm{C}$ for $10 \mathrm{~min}$, which ensures the uniform distribution of graphene on the surface of absorbent paper. Next, putting obtained graphene/paper and $\mathrm{Mg}$ sheet side by side on the air-permeable. Finally, attaching a thin cotton layer containing $\mathrm{KCl}$ powders onto electrodes to form the SAB.

\section{Fabrication of microelectronics and multiplexed biosensor patch}

Pattern and circuit were fabricated by flexible printed circuit board processing techniques of copper (thickness $10 \mu \mathrm{m}$ ) plated with gold (thickness $50 \mathrm{~nm}$ ) on PI substrate. Then, an insulating layer was covered onto the exposing circuit for preventing short circuit. All components, including microcontroller (MSP430FR5969, TI), NFC receiver (NT3H2211, NXP), EEPROM (M24M02-DR, STMicroelectronics), and LDO (TPS76933, TI) were all bonded and electrically connected by Low-temperature solder joints all of the components.

For the fabrication of the multiplexed biosensors patch, the electrode substrate was obtained by the similar process with microelectronics. The top two Au electrodes could be used as the impedance sensor. For the preparation of $\mathrm{Na}^{+}$sensor, PEDOT:PSS was the first layer needed to 
electrodeposited on the Au electrode, which was obtained by constant current electrodeposition $(0.2515 \mathrm{~mA}, 1800 \mathrm{~s})$ in the mixed solution of $0.01 \mathrm{M}$ 3,4-Ethylenedioxythiophene (EDOT) and $0.1 \mathrm{M}$ polystyrene sulfonate (NaPSS). And then $5 \mu \mathrm{L} \mathrm{Na}{ }^{+}$sensitive cocktail was drop-casted on the PEDOT:PSS layer and dried overnight in a $4{ }^{\circ} \mathrm{C}$ refrigerator. To prepare the $\mathrm{Na}^{+}$sensitive cocktail, $100 \mathrm{mg}$ mixture of $\mathrm{Na}$ ionophore $X(1 \% \mathrm{w} / \mathrm{w})$, sodium tetrakis [3,5-bis(trifluoromethyl)phenyl] borate (Na-TFPB, $0.55 \% \mathrm{w} /$ $\mathrm{w})$, polyvinyl chloride (PVC, $K$-value $72-1,33 \% \mathrm{w} / \mathrm{w}$ ), and bis(2-ethylehexyl) sebacate (DOS, $65.45 \% \mathrm{w} / \mathrm{w}$ ) was dissolved in $610 \mu \mathrm{L}$ of tetrahydrofuran 17 . For the $\mathrm{pH}$ sensor, the sensitive layer of PANI was obtained by electrodeposition in the mixture solution of $0.1 \mathrm{M}$ aniline and $1 \mathrm{M} \mathrm{H}_{2} \mathrm{SO}_{4}$ through 50 cycles of cyclic voltammetry $(-0.2$ to $1 \mathrm{~V}$ at a scan rate of $\left.0.1 \mathrm{~V} \mathrm{~s}^{-1}\right)$. For the fabrication of $\mathrm{Ag} / \mathrm{AgCl}$ reference electrode, an Ag layer $(100 \mathrm{~nm})$ was firstly sputtering on the Au electrode. And then, $10 \mu \mathrm{L} 0.1 \mathrm{M}$ $\mathrm{FeCl}_{3}$ was drop-casted onto the $\mathrm{Ag}$ for $30 \mathrm{~s}$ to form $\mathrm{Ag} / \mathrm{AgCl}$. For the reference electrode of $\mathrm{Na}^{+}$sensor, $5 \mu \mathrm{L}$ extra polyvinyl butyral (PVB) cocktail $(79.1 \mathrm{mg}$ of PVB, $50 \mathrm{mg}$ of $\mathrm{NaCl}, 1 \mathrm{mg}$ of block polymer PEO-PPOPEO, and $0.2 \mathrm{mg}$ of carbon nanotubes in $1 \mathrm{ml}$ of methanol) was dropcasted on the $\mathrm{Ag} / \mathrm{AgCl}$ electrode.

\section{Characterization}

The SEM images were obtained by FEI Quanta 250 . The open circuit was measured by the data acquisition (DAQ)/multimeter system (PowerLab 16/35, AD Instruments). Short circuit current was calculated by measuring the voltage of a $2.5 \Omega$ resistance as the load. Polarization curve measurement, chronopotentiometry, constant current electrodeposition, and CV were achieved by the electrochemical work station of $\mathrm{CHI} 660 \mathrm{E}$. The current density was calculated through the reaction area of electrodes $\left(3 \mathrm{~cm}^{2}\right)$ and the power density was the product of voltage and current density. The battery capacity was the product of discharging current and discharging time.

\section{Ethical information for studies involving human subjects}

All experiments involving human subjects were approved by Research Committee of City University of Hong Kong and conducted in compliance with the guidelines. All participants for the studies were fully voluntary and submitted the written informed consents to take part in the study. The device is attached on the subjects' body and thus the necessary but limited identifiable images must be used. All identifiable information was totally consented by the user.

\section{DATA AVAILABILITY}

All data are contained within the manuscript. Raw data are available from the corresponding authors upon reasonable request.

Received: 20 October 2021; Accepted: 11 January 2022; Published online: 08 February 2022

\section{REFERENCES}

1. Deng, J. et al. Electrical bioadhesive interface for bioelectronics. Nat. Mater. 20, 229-236 (2021)

2. Liu, C. et al. Wireless, skin-interfaced devices for pediatric critical care: Application to continuous, noninvasive blood pressure monitoring. Adv. Health. Mater. 10, 2100383 (2021).

3. Zhao, $\mathrm{H}$. et al. Compliant $3 \mathrm{D}$ frameworks instrumented with strain sensors for characterization of millimeter-scale engineered muscle tissues. Proc. Natl Acad. Sci. USA 118, 19 (2021).

4. Chun, K. S. et al. A skin-conformable wireless sensor to objectively quantify symptoms of pruritus. Sci. Adv. 7, eabf9405 (2021).

5. Yu, X. et al. Skin-integrated wireless haptic interfaces for virtual and augmented reality. Nature 575, 473-479 (2019).

6. Bandodkar, A. J. et al. Battery-free, skin-interfaced microfluidic/electronic systems for simultaneous electrochemical, colorimetric, and volumetric analysis of sweat. Sci. Adv. 5, eaav3294 (2019).

7. Song, E. et al. Miniaturized electromechanical devices for the characterization of the biomechanics of deep tissue. Nat. Biomed. Eng. 5, 759-771 (2021).

8. Liu, Y. et al. Electronic skin from high-throughput fabrication of intrinsically stretchable lead zirconate titanate elastomer. Research 2020, 1085417 (2020).

9. Li, D. et al. Miniaturization of mechanical actuators in skin-integrated electronics for haptic interfaces. Microsyst. Nanoeng. 7, 85 (2021).
10. Wong, T. H. et al. Tattoo-like epidermal electronics as skin sensors for humanmachine interfaces. Soft Sci. 1, 10 (2021).

11. Li, D., Yao, K., Gao, Z., Liu, Y. \& Yu, X. Recent progress of skin-integrated electronics for intelligent sensing. Light.: Adv. Manuf. 2, 1-20 (2021).

12. Gao, W. et al. Fully integrated wearable sensor arrays for multiplexed in situ perspiration analysis. Nature 529, 509-514 (2016).

13. Oncescu, V., O'Dell, D. \& Erickson, D. Smartphone based health accessory for colorimetric detection of biomarkers in sweat and saliva. Lab Chip 13, 3232-3238 (2013).

14. Kim, J., Campbell, A. S., de Avila, B. E. F. \& Wang, J. Wearable biosensors for healthcare monitoring. Nat. Biotechnol. 37, 389-406 (2019).

15. Smith, T. W. Blood, sweat, and tears: Exercise in the management of mental and physical health problems. Clin. Psychol.-Sci. Pr. 13, 198-202 (2006).

16. Huang, $X$. et al. Epidermal self-powered sweat sensors for glucose and lactate monitoring. Bio-Des. Manuf. 5, 201-209 (2022).

17. Bandodkar, A. J. et al. Sweat-activated biocompatible batteries for epidermal electronic and microfluidic systems. Nat. Electron. 3, 554-562 (2020).

18. Bandodkar, A. J. et al. Epidermal tattoo potentiometric sodium sensors with wireless signal transduction for continuous non-invasive sweat monitoring. Biosens. Bioelectron. 54, 603-609 (2014).

19. $\mathrm{He}, \mathrm{W}$. et al. Integrated textile sensor patch for real-time and multiplex sweat analysis. Sci. Adv. 5, eaax0649 (2019).

20. Sonner, Z. et al. The microfluidics of the eccrine sweat gland, including biomarker partitioning, transport, and biosensing implications. Biomicrofluidics 9, 031301 (2015).

21. Koh, A. et al. A soft, wearable microfluidic device for the capture, storage, and colorimetric sensing of sweat. Sci. Transl. Med. 8, 366ra165 (2016)

22. Choi, J. et al. Skin-interfaced microfluidic systems that combine hard and soft materials for demanding applications in sweat capture and analysis. Adv. Health. Mater. 10, 4 (2021)

23. Wang, L., Zhang, Y., Pan, J. \& Peng, H. S. Stretchable lithium-air batteries for wearable electronics. J. Mater. Chem. A 4, 13419-13424 (2016).

24. Liu, Z. X. et al. Towards wearable electronic devices: A quasi-solid-state aqueous lithium-ion battery with outstanding stability, flexibility, safety, and breathability. Nano Energy 44, 164-173 (2018).

25. $\mathrm{Pu}, \mathrm{X}$. et al. A self-charging power unit by integration of a textile triboelectric nanogenerator and a flexible lithium-ion battery for wearable electronics. Adv. Mater. 27, 2472-2478 (2015).

26. Zhao, K., Yang, Y., Liu, X. \& Wang, Z. L. Triboelectrification-enabled self-charging lithium-ion batteries. Adv. Energy Mater. 7, 21 (2017).

27. Bae, J. et al. Fiber supercapacitors made of nanowire-fiber hybrid structures for wearable/flexible energy storage. Angew. Chem. Int. Ed. 50, 1683-1687 (2011).

28. Gopi, C. V. V. M., Vinodh, R., Sambasivam, S., Obaidat, I. M. \& Kim, H. J. Recent progress of advanced energy storage materials for flexible and wearable supercapacitor: From design and development to applications. J. Energy Storage 27, 101035 (2020).

29. Sun, J. F. et al. Recent progress of fiber-shaped asymmetric supercapacitors. Mater. Today Energy 5, 1-14 (2017).

30. Huang, X. et al. Wearable biofuel cells based on the classification of enzyme for high power outputs and lifetimes. Biosens. Bioelectron. 124-125, 40-52 (2019).

31. Pan, S. et al. Wearable solar cells by stacking textile electrodes. Angew. Chem. Int. Ed. Engl. 53, 6110-6114 (2014).

32. Yin, S., Jin, Z. \& Miyake, T. Wearable high-powered biofuel cells using enzyme/ carbon nanotube composite fibers on textile cloth. Biosens. Bioelectron. 141 111471 (2019).

33. Liu, Y. M., Wang, L. Y., Zhao, L., Yu, X. G. \& Zi, Y. L. Recent progress on flexible nanogenerators toward self-powered systems. InfoMat 2, 318-340 (2020).

34. Yu, Y. et al. Biofuel-powered soft electronic skin with multiplexed and wireless sensing for human-machine interfaces. Sci. Robot. 5, eaaz7946 (2020).

35. Tan, P., Zou, Y., Fan, Y. \& Li, Z. Self-powered wearable electronics. Wearable Technol. 1, E5 (2020).

36. Wu, M. et al. Self-powered skin electronics for energy harvesting and healthcare monitoring. Mater. Today Energy 21, 100786 (2021).

37. Wu, M. et al. Thin, soft, skin-integrated foam-based triboelectric nanogenerators for tactile sensing and energy harvesting. Mater. Today Energy 20, 100657 (2021).

38. Jeerapan, I., Sempionatto, J. R. \& Wang, J. On-body bioelectronics: wearable biofuel cells for bioenergy harvesting and self-powered biosensing. Adv. Funct. Mater. 30, 1906243 (2020).

39. $\mathrm{Wu}, \mathrm{H}$. et al. Enhanced power generation from the interaction between sweat and electrodes for human health monitoring. ACS Energy Lett. 5, 3708-3717 (2020).

40. Zhang, T., Tao, Z. \& Chen, J. Magnesium-air batteries: From principle to application. Mater. Horiz. 1, 196-206 (2014).

41. Li, C. S., Sun, Y., Gebert, F. \& Chou, S. L. Current progress on rechargeable magnesium-air battery. Adv. Energy Mater. 7, 1700869 (2017)

42. Chawla, N. Recent advances in air-battery chemistries. Mater. Today Chem. 12, 324-331 (2019). 
43. Byon, H. R., Suntivich, J. \& Shao-Horn, Y. Graphene-based non-noble-metal catalysts for oxygen reduction reaction in acid. Chem. Mater. 23, 3421-3428 (2011).

44. Ma, J. L. et al. Effect of functionalized graphene on performance of magnesium/ air battery. Mater. Res Express 6, 085528 (2019).

45. Kim, H. \& Ahn, J. H. Graphene for flexible and wearable device applications. Carbon 120, 244-257 (2017).

46. Oh, Y. S. et al. Battery-free, wireless soft sensors for continuous multi-site measurements of pressure and temperature from patients at risk for pressure injuries. Nat. Commun. 12, 5008 (2021).

47. Song, Y. et al. Wireless battery-free wearable sweat sensor powered by human motion. Sci. Adv. 6, eaay9842 (2020).

48. Park, Y. et al. Wireless, skin-interfaced sensors for compression therapy. Sci. Adv. 6, eabe1655 (2020).

49. Li, L. \& Manthiram, A. Dual-electrolyte lithium-air batteries: Influence of catalyst, temperature, and solid-electrolyte conductivity on the efficiency and power density. J. Mater. Chem. A. 1, 5121-5127 (2013).

50. Bariya, M., Nyein, H. Y. Y. \& Javey, A. Wearable sweat sensors. Nat. Electron. 1 , 160-171 (2018).

51. Nyein, H. Y. Y. et al. A wearable electrochemical platform for noninvasive simultaneous monitoring of $\mathrm{Ca}^{2+}$ and pH. ACS Nano 10, 7216-7224 (2016).

52. Gutruf, P. et al. Wireless, battery-free, fully implantable multimodal and multisite pacemakers for applications in small animal models. Nat. Commun. 10, 5742 (2019).

53. Hajiaghajani, A. et al. Textile-integrated metamaterials for near-field multibody area networks. Nat. Electron. 4, 808-817 (2021).

\section{ACKNOWLEDGEMENTS}

This work was supported by City University of Hong Kong (Grants No. 9667199, 9667221, 9680322), Research Grants Council of the Hong Kong Special Administrative Region (Grant No. 21210820, 11213721), Shenzhen Science and Technology Innovation Commission (Grant No. JCYJ20200109110201713).

\section{AUTHOR CONTRIBUTIONS}

X.H., Y.L., and J.Z. contributed equally to this work. X.H., Y.L., and X.Y. conceived the ideas and designed the experiments. X.H., Y.L., S.N., T.W., H.L., Y.H., and X.Y. wrote the manuscript. X.H., Y.L., J.Z., C.Y., W.P., J.L., L.Z., J.S., and K.Y. performed experiments, X.H., Y.L., Z.G., M.W., D.L., J.L., and R.S. analyzed the experimental data. J.Z., C.Y., and W.P. designed the electrical circuits.

\section{COMPETING INTERESTS}

The authors declare no competing interests.

\section{ADDITIONAL INFORMATION}

Supplementary information The online version contains supplementary material available at https://doi.org/10.1038/s41528-022-00144-0.

Correspondence and requests for materials should be addressed to Xinge $\mathrm{Yu}$.

Reprints and permission information is available at http://www.nature.com/ reprints

Publisher's note Springer Nature remains neutral with regard to jurisdictional claims in published maps and institutional affiliations.

(C) Open Access This article is licensed under a Creative Commons Attribution 4.0 International License, which permits use, sharing, adaptation, distribution and reproduction in any medium or format, as long as you give appropriate credit to the original author(s) and the source, provide a link to the Creative Commons license, and indicate if changes were made. The images or other third party material in this article are included in the article's Creative Commons license, unless indicated otherwise in a credit line to the material. If material is not included in the article's Creative Commons license and your intended use is not permitted by statutory regulation or exceeds the permitted use, you will need to obtain permission directly from the copyright holder. To view a copy of this license, visit http://creativecommons. org/licenses/by/4.0/.

(c) The Author(s) 2022 